PAPER

\title{
Evidence for grey matter MTR abnormality in minimally disabled patients with early relapsing-remitting multiple sclerosis
}

\author{
G R Davies, L Ramió-Torrentà, A Hadjiprocopis, D T Chard, C M B Griffin, W Rashid, G J Barker, \\ R Kapoor, A J Thompson, D H Miller
}

J Neurol Neurosurg Psychiatry 2004;75:998-1002. doi: 10.1136/jnnp.2003.021915

See end of article for authors' affiliations

.....................

Correspondence to: Professor D H Miller, NMR Research Unit, Institute of Neurology, Queen Square, London WCIN 3BG, UK; d.miller@ ion.ucl.ac.uk

Received 30 June 2003 Revised

10 September 2003

Accepted

1 November 2003

\begin{abstract}
Objectives: To establish whether magnetisation transfer ratio (MTR) histograms are sensitive to change in normal appearing grey matter (NAGM) in early relapsing-remitting multiple sclerosis (RRMS) in the absence of significant disability; and to assess whether grey or white matter MTR measures are associated with clinical measures of impairment in early RRMS

Methods: 38 patients were studied (mean disease duration 1.9 years (range 0.5 to 3.7); median expanded disability status scale (EDSS) 1.5 (0 to 3)), along with 35 healthy controls. MTR was determined from proton density weighted images with and without MT presaturation. SPM99 was used to generate normal appearing white matter (NAWM) and NAGM segments of the MTR map, and partial voxels were minimised with a 10 pu threshold and voxel erosions. Mean MTR was calculated from the tissue segments. Atrophy measures were determined using a 3D fast spoiled gradient recall sequence from 37 patients and 17 controls.

Results: Mean NAGM and NAWM MTR were both reduced in early RRMS (NAGM MTR: 31.9 pu in patients $v 32.2 \mathrm{pu}$ in controls; $p<0.001$; NAWM MTR: 37.9 v $38.3 \mathrm{pu}, \mathrm{p}=0.001$ ). Brain parenchymal fraction (BPF) correlated with NAGM MTR, but when BPF was included as a covariate NAGM MTR was still lower in the patients $(p=0.009)$. EDSS correlated with NAGM MTR $(r=0.446 p=0.005)$.

Conclusions: In early RRMS, grey matter MTR abnormality is apparent. The correlation with mild clinical impairment (in this essentially non-disabled cohort) suggests that NAGM MTR could be a clinically relevant surrogate marker in therapeutic trials.
\end{abstract}

potential as surrogate markers in therapeutic trials, particularly as lesion load measures have been shown to correlate only modestly with future disability. ${ }^{11}{ }^{12}$ Intrinsic grey matter abnormality may be of special interest as grey matter atrophy occurs early in multiple sclerosis ${ }^{5}$ and may be better associated with clinical status than white matter changes. ${ }^{7}$

This study builds on the earlier work of Griffin et al, ${ }^{3}$ by imaging a larger cohort of non-disabled early RRMS patients and, rather than analysing small ROIs, MTR measurements have been achieved using a tissue segmentation technique that allows a better assessment of global but tissue specific MTR changes in early multiple sclerosis.

Our aims in the study were, first, to establish whether MTR histograms are sensitive to NAGM change in early RRMS in the absence of significant disability, and second, to assess whether grey matter or white matter MTR measures were associated with clinical measures of impairment in early RRMS.

\section{METHODS}

\section{Subjects}

The study was approved by the joint ethics committee of the Institute of Neurology and the National Hospital for Neurology and Neurosurgery, Queen Square, London. Written informed consent was obtained from all subjects.

Abbreviations: BPF, brain parenchymal fraction; EDSS, expanded disability status scale; GM, grey matter; GMF, grey matter fraction; MSFC, multiple sclerosis functional composite (score); MTR, magnetisation transfer ratio; NAGM, normal appearing grey matter; NAWM, normal appearing white matter; ROI, region of interest; RRMS, relapsing-remitting multiple sclerosis; $\mathrm{Tl}$, total intracranial volume; $\mathrm{WM}$, white matter; WMF, white matter fraction

MR measures that detect normal appearing tissue abnormality before the onset of permanent disability may have 
MTR and lesion load measures

We studied 38 patients and 35 controls. Patients had clinically definite early RRMS ${ }^{13}{ }^{14}$ ( 28 female, 10 male; mean age 36.3 years (range 24 to 56); disease duration at baseline 1.9 years ( 0.5 to 3.7$)$; median expanded disability status scale $(\text { EDSS })^{15} 1.5(0$ to 3$\left.)\right)$. Controls were healthy volunteers (19 female, 16 male; mean age 38.5 years (23 to 59). Patients were not on disease modifying drugs when imaged. EDSS scores and multiple sclerosis functional composite (MSFC) scores ${ }^{16}$ were determined in all patients. The patients were not significantly disabled (by definition, EDSS $\leqslant 3$ ) but they did have a range of milder clinical impairments that were quantified using EDSS and MSFC.

\section{Atrophy measures}

A subset of those imaged with the MT sequence also underwent three dimensional imaging of the brain, allowing brain volume determination. We studied 37 of the patients with this additional sequence ( 28 female, nine male; mean age 36.1 years (24 to 56 ); disease duration 1.8 years $(0.5$ to 3.7); median EDSS score 1.5 (0 to 3)), along with 17 of the healthy controls ( 10 female, seven male; mean age 35.9 years (30 to 54$)$ ).

\section{MRI acquisition}

Imaging was undertaken on a 1.5 Tesla Signa scanner (General Electric, Milwaukee, Wisconsin, USA).The two dimensional dual echo interleaved spin echo (SE) MTI sequence has been described previously ${ }^{17}$ and was acquired in all controls and multiple sclerosis subjects. Repetition time (TR) was $1500 \mathrm{~ms}$ with echo times (TE) of 19/90 ms. Images were acquired with a $128 \times 256$ matrix over a $24 \mathrm{~cm}$ field of view (FOV) and reconstructed as a $256 \times 256$ matrix. Twenty eight contiguous $5 \mathrm{~mm}$ axial slices covering the whole brain were acquired both with and without presaturation. The saturating MT pulse was $64 \mathrm{~ms}$ in duration and $2 \mathrm{kHz}$ offwater resonance. The power of the MT pulse was $14.6 \mu \mathrm{T}$, with a flip angle of $1430^{\circ}$. Saturated and unsaturated images were interleaved, resulting in inherently registered saturated and unsaturated datasets. MTR was calculated from the short echo images on a pixel by pixel basis according to $\left\{\left[\mathrm{M}_{\mathrm{o}}-\mathrm{M}_{\mathrm{s}}\right] /\right.$ $\left.\left[M_{o}\right]\right\} \times 100$ per cent units (pu), where $M_{s}$ and $M_{o}$ represent the signal intensities with and without the saturation pulse, respectively. In a subset of subjects, a three dimensional, inversion prepared, fast spoiled gradient recall (3D FSPGR) sequence (TR $16 \mathrm{~ms}$, TE $4.2 \mathrm{~ms}$, inversion time $450 \mathrm{~ms}$, matrix $256 \times 160$, FOV $300 \times 225 \mathrm{~mm}$ (interpolated during reconstruction to a final in-plane resolution of $1.2 \times 1.2 \mathrm{~mm}$ ), NEX 1, with $124 \times 1.5 \mathrm{~mm}$ slices covering the whole brain) was also acquired during the same scanning session. The acquisition time was 30 minutes.

\section{Image post processing}

MTR and lesion load analysis

Lesions were contoured using Dispimage (Plummer, Department of Medical Physics and Bioengineering, UCL, London, UK) on the unsaturated proton density weighted images, according to previously defined rules. ${ }^{18}$ Segmentation of the T2 weighted images into grey matter, white matter, and CSF segments was carried out using SPM99 (Wellcome Department of Cognitive Neurology, UK). A whole brain mask (excluding CSF and other non-brain parenchyma) was generated in SPM99 and then applied to the (inherently registered) calculated MTR map. A maximum likelihood algorithm, using the grey and white matter probability outputs from SPM99, was then employed to segment the MTR whole brain map into white and grey matter segments. (Voxels were determined as grey matter or white matter, depending on which probability map showed the greatest probability at that location.) Lesions were excluded from the analysis by setting their pixel value to zero, leaving only normal appearing white matter (NAWM) and normal appearing grey matter (NAGM). Partial volume voxels were minimised with a $10 \mathrm{pu}$ threshold and two successive erosions of white matter and a single erosion of the grey matter (two erosions of grey matter resulted in too much being eliminated) (fig 2). Normalised NAWM, NAGM, and whole white matter histograms were generated with a bin width of $0.1 \mathrm{pu}$ and a smoothing window of $\pm 0.3 \mathrm{pu}$. The mean MTR values for NAWM and NAGM were calculated from these histograms.

\section{Atrophy measures}

Brain volume measurements were determined using the 3D FSPGR data which were processed in SPM99 to generate probability maps as described above. Brain stem and spinal cord were excluded from the analysis by visually determining the slice immediately caudal to the cerebellum. Volumes were then estimated using in-house software. Lesion masks were derived (using lesion segmentations carried out manually by a previously described technique ${ }^{6}$ ) and voxels lying within these masks were defined as lesional tissue. All other voxels were then defined as grey matter (GM), white matter (WM), cerebrospinal fluid (CSF), or other tissue, depending upon which probability map showed the greatest probability at that location. The total intracranial volume (TI) was determined by adding the GM, WM, and CSF volumes. Brain parenchymal fraction (BPF) was then calculated as [GM+WM+lesion volume]/TI. The grey matter fraction (GMF) and white matter fraction (WMF) were also determined by calculating GM/TI and [WM+lesion volume]/TI, respectively.

\section{Validation of segmentation technique in the presence of focal white matter lesions}

As the presence of lesions may have an effect on SPM99 tissue segmentation, and thus differentially affect the results for patients and controls, an assessment of the degree of lesion associated bias was made. In all controls, 60 artificial lesions (uniform cylindrical volumes, each $0.25 \mathrm{ml}$ ) were placed within white matter, providing a simulated lesion volume of $15 \mathrm{ml}$ per subject. This corresponded to the average lesion volume observed in the multiple sclerosis subjects. The signal intensity of artificial lesions was set at a value that appears to have the most marked effect upon SPM99 segmentation (midway between grey matter and CSF signal intensity). The average change in mean white matter MTR and mean grey matter MTR was $+0.03 \mathrm{pu}$ and $-0.09 \mathrm{pu}$, respectively. The effect was insufficient to account for any differences subsequently seen between patients and control subjects.

\section{Statistical analysis}

MTR and atrophy were compared between patients and controls using linear modelling (SPSS 11.0). Sex and diagnosis (multiple sclerosis or control) were included as fixed factors, with age as a continuous covariate. This approach was prospectively defined on the basis of the age and sex imbalances between patients and controls. Associations between clinical variables and MTR were estimated using Spearman's rank correlation test.

\section{RESULTS Imaging}

Average T2 lesion load in the patients was $14.9 \mathrm{ml}$ (mean) and $12.4 \mathrm{ml}$ (median) (range 2.1 to $47.7 \mathrm{ml}$ ).

Mean (SD) NAWM MTR in the patients was 37.9 (0.5) pu, $v 38.3(0.4) \mathrm{pu}$ in the controls. Linear modelling showed a significant disease effect on NAWM MTR $(p=0.001)$ but no 


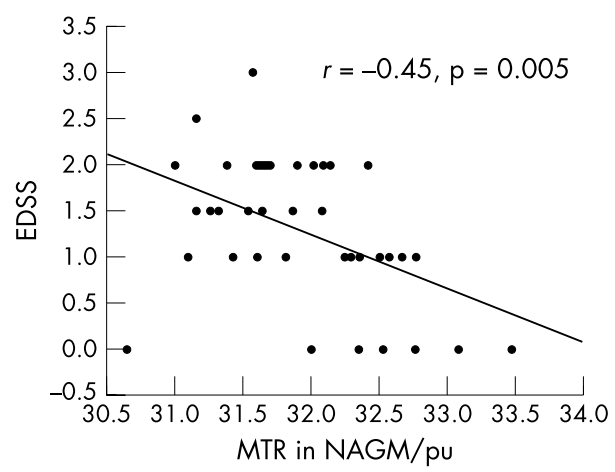

Figure 1 Inverse correlation between expanded disability status scale (EDSS) and normal appearing grey matter (NAGM) magnetisation transfer ratio (MTR).

effect for either age or sex (table 1). Mean NAGM MTR in the patients was $31.9(0.6) \mathrm{pu}, v 32.3(0.5) \mathrm{pu}$ in the controls. Linear modelling showed a significant disease effect on NAGM MTR $(p<0.001)$ and also a significant effect of sex $(\mathrm{p}<0.001)$, with female subjects having higher grey matter MTR than male subjects. No effect of age on NAGM MTR was observed (table 1).

T2 lesion load was inversely correlated with NAGM (Spearman's correlation coefficient $(r)=-0.56 \mathrm{p}<0.001$ ) and NAWM MTR $(r=-0.53, \mathrm{p}=0.001)$. In the patients, NAGM MTR was correlated with NAWM MTR $(r=0.43$, $\mathrm{p}=0.008)$. In the controls only a trend for an association between NAGM MTR and NAWM MTR was seen $(r=0.33$, $\mathrm{p}=0.06$ ).

Volume measures derived from the 124 slice three dimensional dataset (a subset of the cohort) showed evidence of increased whole brain and WM atrophy in the multiple sclerosis group. Mean (SD) BPF was 0.810 (0.026) in the patients $v 0.835(0.28)$ in controls (linear model disease effect: $\mathrm{p}<0.001)$. WMF was $0.263(0.017)$ in patients $v 0.282$ (0.012) in controls (linear model disease effect term: $\mathrm{p}<0.001)$. GMF was $0.547(0.023)$ in patients $v 0.552$ $(0.023)$ in controls (linear model disease effect term: nonsignificant $(\mathrm{p}=0.18))$. BPF was correlated with NAGM MTR $(r=0.69, \mathrm{p}<0.001)$ but not with NAWM MTR $(r=0.21$, $\mathrm{p}=0.21)$. The effect of atrophy on NAGM MTR was therefore assessed by including BPF as a covariate in the linear model (with sex as a fixed factor). This showed that the disease effect on NAGM MTR remained significant $(p=0.009)$.

\section{Clinical status}

Median EDSS in the patients was 1.5 (range 0 to 3 ). EDSS was inversely correlated with NAGM MTR $(r=-0.45$ $\mathrm{p}=0.005) \quad$ (fig 2) but not with NAWM MTR $(r=-0.2$, $\mathrm{p}=0.9)$. BPF was inversely related to EDSS $(r=-0.39$, $\mathrm{p}=0.02$ ). Borderline significance was seen for an association

Table 1 Normal appearing white and grey matter magnetisation transfer ratio values in patients and controls

\begin{tabular}{llll}
\hline & $\begin{array}{l}\text { MS subjects } \\
(\mathbf{n}=\mathbf{3 8})\end{array}$ & Controls $(\mathbf{n}=\mathbf{3 5 )}$ & p Value* \\
\hline NAWM MTR & $37.9(0.5) \mathrm{pu}$ & $38.3(0.4) \mathrm{pu}$ & $\mathrm{p}=0.001$ \\
NAGM MTR & $31.9(0.6) \mathrm{pu}$ & $32.3(0.5) \mathrm{pu}$ & $\mathrm{p}<0.001$
\end{tabular}

*Significance of disease effect factor (multiple sclerosis or control) in linear model.

MS, multiple sclerosis; MTR, magnetisation transfer ratio; NAGM, normal appearing grey matter; NAWM, normal appearing white matter. between T2 lesion load and EDSS $(r=0.31, \mathrm{p}=0.06)$. The MSFC score and the three $z$ scores from which the MSFC is derived ( $z$-arm, $z$-cognition, and $z$-leg) were also determined in this cohort. ${ }^{16}$ Borderline evidence for an association was seen between $z$-arm and NAGM MTR $(r=0.31, \mathrm{p}=0.06)$ but no other association was seen between the MSFC or $z$ scores and MTR measures. T2 lesion load was inversely correlated with the MSFC $(r=-0.36, \mathrm{p}=0.03)$ and was also correlated with the $z$-leg score $(r=0.44, \mathrm{p}=0.005)$. (More negative values correspond to greater disability with the $z$-leg score, but the converse is true with the MSFC.) Borderline evidence was seen for an inverse association between disease duration and NAWM MTR $(r=-0.29, \mathrm{p}=0.08)$ but not with NAGM MTR.

\section{DISCUSSION}

We detected NAGM and NAWM MTR abnormalities in RRMS in a cohort who have early disease (mean disease duration 1.9 years) and mild clinical impairments but not major disability. Furthermore, clinical impairments in early RRMS were associated with NAGM abnormality but not with changes in NAWM.

The ability to detect grey matter abnormality with MTR has been shown before in a cohort with established RRMS (mean disease duration five years (range 1 to 15 ), EDSS range 1 to $6)^{4}$ but not in RRMS patients with minimal disability and early disease. Grey matter involvement, however, appears to be a feature of early RRMS and has been found in studies using several other MR measures, including Tl relaxation, spectroscopic metabolites, and atrophy. ${ }^{35}{ }^{5}$ Also, in a recent study of patients with clinically isolated syndromes suggestive of multiple sclerosis, a reduction of 0.3 pu NAGM MTR was observed. ${ }^{8}$

Changes within normal appearing brain tissue in multiple sclerosis could play an important role in the development of disability, particularly as changes in lesion load correlate only modestly with clinical status. ${ }^{11} 12$ MR methods of assessing change within normal appearing tissue may therefore be of use in clinical trials-particularly if such measures are shown to be good predictors of future impairment or disability, as a key requirement of a validated surrogate outcome measure is that it should be associated with a clinically relevant outcome. ${ }^{19}$ An additional valuable feature would be that such measures should be sensitive to normal appearing tissue abnormality early in the course of the disease, before the onset of significant disability.

While NAGM MTR was associated with clinical impairment using the EDSS, T2 lesion load was associated with clinical impairment using the MSFC. Potentially both lesions and NAGM abnormalities contribute to clinical dysfunction, and although they were partly correlated with each other, their effects on clinical function may be different. However, before concluding that such outcome measures have complementary roles in studies of natural history and treatment monitoring, it would be important to study larger cohorts and serial data.

The present study relied upon accurate tissue segmentation and, in particular, on the fact that lesions did not affect voxel classification. In order to quantify this, lesion simulation was undertaken in controls. The effect noted, however, was small and insufficient to account for the MTR changes observed. In addition, the segmentations were independently verified by a rater who was blinded to the clinical details of the subjects, and the segmentations appeared to be anatomically valid.

The effect of partial volume voxels at the CSF-grey matter interface have a proportionally greater effect on the mean grey matter MTR as atrophy occurs. In this cohort, atrophy has been shown to occur (albeit principally within white matter) and therefore it is possible that some of the MTR decrease is partial volume related. As a result, every possible 
attempt has been made to reduce partial volume voxels through the use of a CSF reducing threshold and erosion of outer voxels. One erosion was used in grey matter, as further erosions resulted in only very limited amounts of grey matter remaining. With these steps, it seems more likely that the NAGM MTR abnormality reflects true intrinsic abnormality rather than partial volume effects of atrophy. Partial volume voxels at the GM-WM interface and the WM-CSF interface may also have an effect on MTR, but as erosions were applied at the inner surface (as well as the outer) these partial volume voxels will also have been minimised.

In further support of NAGM MTR abnormality being secondary to intrinsic tissue pathology rather than atrophy per se, BPF was included as a covariate in the statistical analysis. Such an analysis is conservative as it is liable not only to eliminate MTR differences secondary to partial volume artefact but also to eliminate intrinsic MTR differences that are associated with atrophy. Nonetheless, when this analysis was undertaken, differences were still observed between patient and control NAGM MTR.

The atrophy noted in this cohort appears to be secondary principally to white matter volume change, although GM volume in the patients was lower (albeit non-significantly) than in the controls. A previous study ${ }^{6}$ did show GM atrophy in early RRMS, and our inability to detect it here might result from the fact that we had fewer controls.

A further concern is that juxtacortical lesions and perilesional tissue may have been erroneously classified as grey

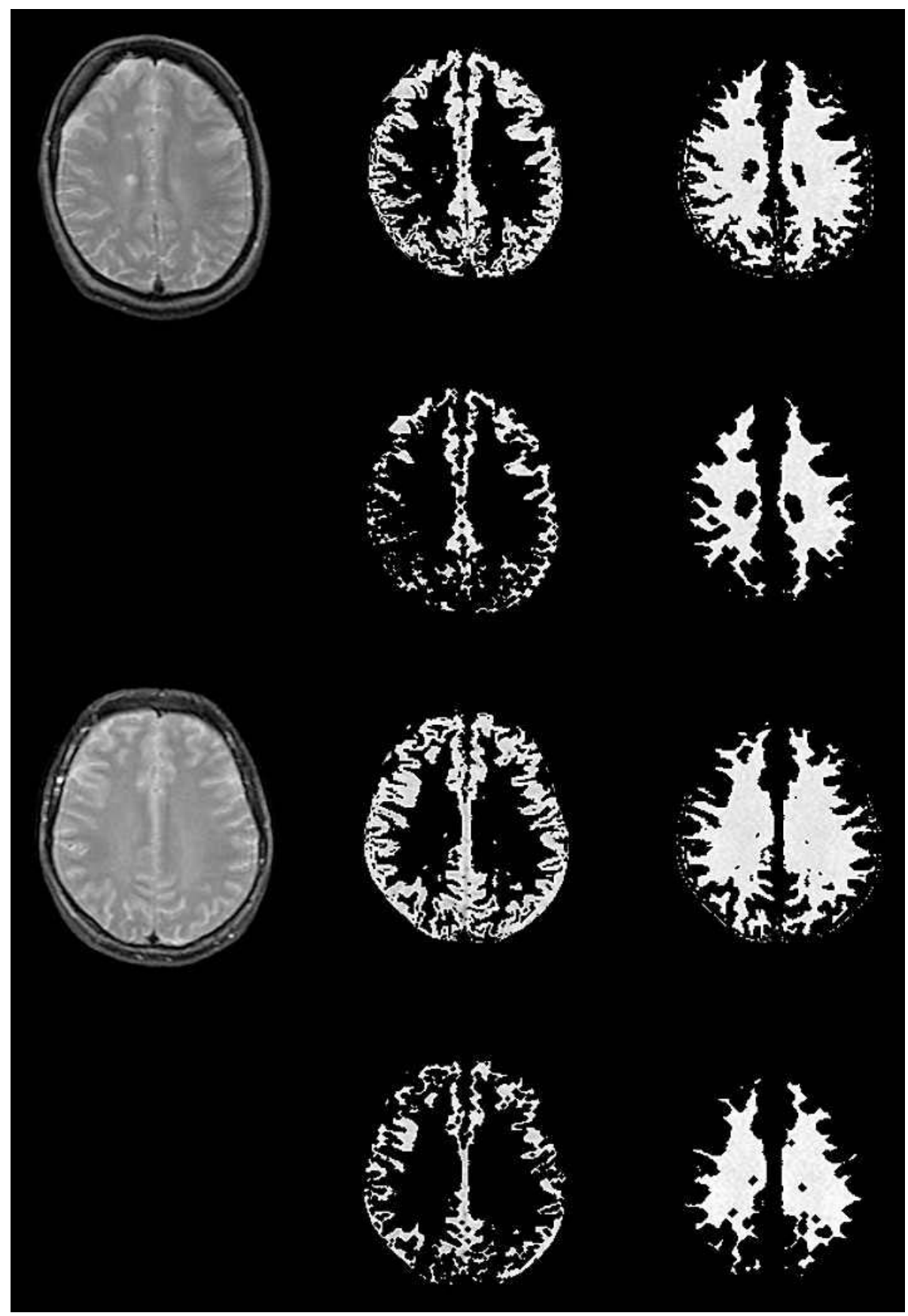

Figure 2 Images from a patient with multiple sclerosis (top two rows) and a healthy control (lower two rows). Left column: T2 weighted images. Central column: grey matter segments of the magnetisation transfer ratio (MTR) map (above) subjected to a 10 pu threshold and one erosion (below). Right column: white matter segments of the MTR map (above) subjected to a 10 pu threshold and two erosions (below). 
matter. However, overt lesions are removed by lesion masking, and subtle areas of abnormality (not detected on proton density weighted imaging) would have MTR values midway between grey matter and white matter. Such areas therefore would not decrease NAGM MTR.

Our statistical analysis was prospectively designed and used linear modelling to account for age and sex imbalances seen between subjects with multiple sclerosis and controls. Where positive disease effects were found, differences between patients and controls were verified by the MannWhitney U test (which proved to be significant, $\mathrm{p}<0.01$ ) for all such results. Corrections for multiple comparisons have not been undertaken (principally as the number of comparisons was not large), and so some of the weaker correlations should be viewed with caution.

What is the pathological basis for the observed normal appearing brain tissue MTR abnormality in early multiple sclerosis? It is known that MTR correlates with axonal and myelin density, ${ }^{9}$ but also, to a certain extent, with inflammatory change. ${ }^{10}$ In NAWM, histopathological findings include gliosis, inflammatory infiltrates, and axonal loss ${ }^{20} 21$ with the latter occurring possibly as a result of Wallerian degeneration of axons transected in lesions. It is notable that acute axonal transection occurs in inflammatory lesions, ${ }^{22}$ more so at an early stage of the disease. ${ }^{23}$ In grey matter, neuronal and myelin loss has been reported in grey matter lesions, which are less inflammatory ${ }^{24}$ and less MR visible than lesions in white matter. ${ }^{25}$ Such changes could account for the MTR abnormalities seen in this study.

\section{Conclusions}

Our study shows that MTR is sensitive to abnormalities in normal appearing grey and white matter in relapsingremitting multiple sclerosis in a cohort of patients with early disease (mean disease duration 1.9 years) and only mild clinical impairment. The correlation of EDSS and grey matter MTR suggests that NAGM MTR abnormality is clinically relevant in early RRMS and therefore may have potential as a surrogate marker in clinical trials. However, these modest correlations are preliminary and so should be confirmed in a larger cohort. In addition, in order to act as a surrogate marker, it is necessary to show that MTR in early multiple sclerosis is sensitive to change over time, and that MTR changes correlate with future disability. If such associations are shown, it would imply that MTR measures could be a clinically relevant outcome for therapeutic interventions.

\section{ACKNOWLEDGEMENTS}

We thank D MacManus, R Gordon, C Benton, and the Multiple Sclerosis Society of Great Britain and Northern Ireland for their continuing support of the NMR Research Unit (including funding GRD, WR, AH, and GJB during the course of this study), Schering AG for support of DTC, and all the people who kindly agreed to take part in this study.

\section{Authors' affiliations}

G R Davies, L Ramió-Torrentà, A Hadjiprocopis, D T Chard, C M B Griffin, W Rashid, R Kapoor, A J Thompson, D H Miller, NMR Research Unit, Institute of Neurology, University College London, Queen Square, London, UK
G J Barker, Neuroimaging Research Group, Institute of Psychiatry, Kings College London, De Crespigny Park, London

Competing interests: none declared

\section{REFERENCES}

1 Dousset V, Grossman RI, Ramer KN, et al. Experimental allergic encephalomyelitis and multiple sclerosis: lesion characterization with magnetization transfer imaging. Radiology 1992;182:483-91.

2 Filippi M, Campi A, Dousset V, et al. A magnetization transfer imaging study of normal-appearing white matter in multiple sclerosis. Neurology 1995;45:478-82.

3 Griffin CM, Chard DT, Parker GJ, et al. The relationship between lesion and normal appearing brain tissue abnormalities in early relapsing remitting multiple sclerosis. J Neurol 2002;249:193-9.

$4 \mathrm{Ge} \mathrm{Y,} \mathrm{Grossman} \mathrm{RI,} \mathrm{Udupa} \mathrm{JK,} \mathrm{et} \mathrm{al.} \mathrm{Magnetization} \mathrm{transfer} \mathrm{ratio} \mathrm{histogram}$ analysis of normal-appearing gray matter and normal-appearing white matter in multiple sclerosis. J Comput Assist Tomogr 2002;26:62-8.

5 Chard DT, Griffin CM, McLean MA, et al. Brain metabolite changes in cortical grey and normal-appearing white matter in clinically early relapsing-remitting multiple sclerosis. Brain 2002;125:2342-52.

6 Chard DT, Griffin CM, Parker GJ, et al. Brain atrophy in clinically early relapsing-remitting multiple sclerosis. Brain 2002;125:327-37.

7 De Stefano N, Matthews PM, Filippi M, et al. Evidence of early cortical atrophy in MS: relevance to white matter changes and disability. Neurology 2003;60:1157-62.

8 Traboulsee A, Dehmeshki J, Brex PA, et al. Normal-appearing brain tissue MTR histograms in clinically isolated syndromes suggestive of MS. Neurology 2002;59:126-8.

9 van Waesberghe JH, Kamphorst W, De Groot CJ, et al. Axonal loss in multiple sclerosis lesions: magnetic resonance imaging insights into substrates of disability. Ann Neurol 1999:46:747-54.

10 Gareau PJ, Rutt BK, Karlik SJ, et al. Magnetization transfer and multicomponent T2 relaxation measurements with histopathologic correlation in an experimental model of MS. J Magn Reson Imaging 2000; 1 1:586-95.

11 Molyneux PD, Barker GJ, Barkhof F, et al. Clinical-MRI correlations in a European trial of interferon beta- $1 \mathrm{~b}$ in secondary progressive MS. Neurology 2001;57:2191-7

12 Paolillo A, Pozzilli C, Giugni E, et al. A 6-year clinical and MRI follow-up study of patients with relapsing-remitting multiple sclerosis treated with interferon-beta. Eur J Neurol 2002;9:645-55.

13 Poser CM, Paty DW, Scheinberg L, et al. New diagnostic criteria for multiple sclerosis: guidelines for research protocols. Ann Neurol 1983;13:227-31.

14 Lublin FD, Whitaker JN, Eidelman BH, et al. Management of patients receiving interferon beta-1b for multiple sclerosis: report of a consensus conference. Neurology 1996;46:12-18.

15 Kurtzke JF. Rating neurologic impairment in multiple sclerosis: an expanded disability status scale (EDSS). Neurology 1983;33:1444-52.

16 Fischer JS, Rudick RA, Cutter GR, et al. The multiple sclerosis functional composite measure (MSFC): an integrated approach to MS clinical outcome assessment. National MS Society Clinical Outcomes Assessment Task Force. Multiple Sclerosis 1999;5:244-50.

17 Barker GJ, Tofts PS, Gass A. An interleaved sequence for accurate and reproducible clinical measurement of magnetization transfer ratio. Magn Reson Imaging 1996;14:403-11.

18 Sailer M, O'Riordan J, Thompson AJ, et al. Quantitative MRI in patients with clinically isolated syndromes suggestive of demyelination. Neurology 1999:52:599-606

19 McFarland HF, Barkhof F, Antel J, et al. The role of MRI as a surrogate outcome measure in multiple sclerosis. Multiple Sclerosis 2002;8:40-51.

20 Allen IV, McKeown SR. A histological, histochemical and biochemical study of the macroscopically normal white matter in multiple sclerosis. J Neurol Sci 1979:41:81-91.

21 Evangelou N, Esiri MM, Smith S, et al. Quantitative pathological evidence for axonal loss in normal appearing white matter in multiple sclerosis. Ann Neurol 2000;47:391-95.

22 Trapp BD, Peterson J, Ransohoff RM, et al. Axonal transection in the lesions of multiple sclerosis. N Engl J Med 1998;338:278-85.

23 Kuhlmann T, Lingfield G, Bitsch A, et al. Acute axonal damage in multiple sclerosis is most extensive in early disease and decreases over time. Brain 2002; 125:2202-12.

24 Peterson JW, Bo L, Mork S, et al. Transected neurites, apoptotic neurons, and reduced inflammation in cortical multiple sclerosis lesions. Ann Neurol 2001; 50:389-400.

25 Kidd D, Barkhof F, McConnell R, et al. Cortical lesions in multiple sclerosis. Brain 1999;122:17-26. 